\title{
Physics-informed machine learning of flow and reactive transport in porous media
}

\author{
VINCENT LIU AND HONGKYU YOON
}

Sandia National Laboratories

Presenting Author: vinliu@sandia.gov

Flow and reactive transport in fractured and porous media is fundamental to understanding coupled multiphysics processes critical to various geoscience and environmental applications such as geologic carbon storage, subsurface energy recovery, and environmental fate and transport. Although a pore-scale model provides fundamental mechanistic explanations of flow and reactive transport processes, pore scale modeling often faces the challenges in computational expense and explicit knowledge of pore structures. Recent emerging machine learning methods with physics-informed constraints can provide a new means to improve computational efficiency while improving model prediction by overcoming overfitting to training data that is typical of conventional machine learning methods. Here we employ physics informed neural network approaches to solve for physical systems while respecting the laws of physics given by general nonlinear differential equations. In this work, we apply physics informed neural networks to predict fluid flow and reactive transport in a constrained geometry and compare our results with analytical and numerical solutions produced by fluid dynamics simulations. We test our models to evaluate various flow and transport problems in 2D domains using the advectiondiffusion and (Navier) Stokes differential equations. Additionally, we test flow and transport problems in the presence of an obstructing cylinder to simulate fluid velocity and concentration distributions from advection-diffusion-reaction. Comparison of results between the physics-informed deep learning approach and fluid dynamics simulations will be discussed to highlight the accuracy and efficiency of physicsinformed neural networks.

SNL is managed and operated by NTESS under DOE NNSA contract DE-NA0003525. 